\title{
论文
}

\section{渗透型高聚物在富水砂层中堵水规律试验与 数值模拟研究}

方宏远 ${ }^{1,2,3^{*}}$, 刘康 ${ }^{1}$, 杜雪明 $1,2,3^{*}$, 王复明 2,3

1. 郑州大学水利科学与工程学院, 郑州 450001;

2. 重大基础设施检测修复技术国家地方联合工程实验室, 郑州 450001;

3. 水利与交通基础设施安全防护河南省协同创新中心, 郑州 450001

*E-mail: 18337192244@163.com; 2007-dxm@163.com

收稿日期: 2021-10-06; 接受日期: 2022-01-27; 网络版发表日期: 2022-08-16

国家自然科学基金(批准号: 52008379, 51678536, 41404096)、国家重点基础研究发展规划(编号: 2017YFC1501204)和国家基础研究计划(编 号: 2015CB060200)资助项目

摘要渗透注浆是修建隧道穿越富水砂层时防渗堵水的重要方法之一。与普通化学浆液不同的是, 该渗透型高聚 物在浆液扩散过程中具有双组分反应并快速固结的特点, 适用于在富水砂层中注浆堵水. 然而由于其扩散过程中 的快速固结的特点，使得其在富水砂层的扩散规律更为复杂。为了研究该渗透型高聚物在富水砂层中的堵水效 果, 制作了一套可视化的富水砂层渗透注浆模型试验装置, 该装置具备可观察浆液扩散距离、监测注浆前后孔隙 水压力变化和测量堵水率等功能. 以堵水率、渗透系数作为评价富水砂层渗透注浆堵水防渗效果的性能指标, 选 取砂层粒径、动水压力、注浆压力和黏性土含量4个因素作为渗透注浆的影响因素, 开展了砂层渗透注浆堵水防 渗效果的正交试验，获得了影响渗透型高聚物浆液在富水砂层渗透注浆效果的主控因素以及各单因素对注浆效 果的影响特征. 同时, 对模型试验进行了数值模拟, 重点分析了注浆过程中的浆-水两相渗流场压力、流速和浆液 扩散的变化过程, 并与试验实测浆液扩散距离进行了对比验证. 研究结果表明: (1) 富水砂层经渗透型高聚物注浆 后, $10 \mathrm{~min}$ 内堵水率可达到 $90 \%$ 以上, $1 \mathrm{~d}$ 渗透系数可达到 $10^{-7} \mathrm{~cm} / \mathrm{s}$ 数量级, 达到了理想的堵水防渗效果; (2) 在一定 注浆压力范围内，注浆压力是控制富水砂层注浆堵水防渗效果的主控因素，随着注浆压力的增大，10 min内堵水 率逐渐增大且砂层 $1 \mathrm{~d}$ 渗透系数显著降低; (3) 反应结束后, 浆液能扩散到的砂层位置处, 孔隙水压力接近 0 ; (4) 数 值模拟结果表明, 浆-水两相渗流压力场从注浆孔向出水边界递减, 顺水段渗流速度受水流冲刷明显大于逆水段, 浆液存在逆水扩散现象, 扩散距离的模拟结果与固结体实测结果误差不大; (5) 通过多元线性回归分析, 得到注浆 堵水率、固结体 $1 \mathrm{~d}$ 的渗透系数与动水压力、注浆压力、砂层粒径、黏性土含量的回归方程.

关键词富水砂层, 渗透注浆, 渗透型高聚物, 模型试验, 数值模拟, 堵水

引用格式: 方宏远, 刘康, 杜雪明, 等. 渗透型高聚物在富水砂层中堵水规律试验与数值模拟研究. 中国科学: 技术科学, 2023, 53: 457-472 Fang H Y, Liu K, Du X M, et al. Experiment and numerical simulation of water plugging law of permeable polymer in the water-rich sand layer (in Chinese). Sci Sin Tech, 2023, 53: 457-472, doi: 10.1360/SST-2021-0461 


\section{1 引言}

隧道施工经常遇到富水软弱砂层，在隧道建设使 用过程中极易诱发涌水溃砂的灾害 ${ }^{[1,2]}$, 严重威胁到隧 道施工和使用过程中的安全。注浆技术是隧道施工穿 越砂层段堵水防渗的重要方法之一，通过注浆可一定 程度地提高砂层的整体力学性能和抗渗性能 ${ }^{[3,4]}$. 水泥 浆液或普通化学浆液作为传统注浆材料的代表被广泛 应用于注浆加固中 ${ }^{[5]}$, 然而, 由于富水砂层中水流的作 用, 该注浆材料浆液容易被稀释或冲散, 很难达到理想 的注浆效果. 近年来, 以高聚物为代表的注浆材料逐渐 被人们熟知并应用于一些大小工程中 ${ }^{[6]}$, 由于高聚物 浆液自身特性，使得其扩散过程比普通浆液更具有复 杂性，如何利用高聚物注浆材料改善富水砂层的工程 特性已成为急需解决的关键问题之一.

目前国内外众多学者对注浆堵水防渗问题进行了 相关研究. Du等人 ${ }^{[7]}$ 通过模型试验和工程试验得到了 特征裂隙参数和水的流速对水泥浆液在有流水的裂隙 中的扩散规律; 刘健等人 ${ }^{[8]}$ 通过模型试验和数值模拟 系统研究了水泥浆液在动水条件下平面裂隙中的扩散 规律; 刘强等人 ${ }^{[9]}$ 利用室内模型试验对水下隧道的渗 流场进行分析，得到了隧道在不同排水方式下注浆圈 的分布规律; 张连震等人 ${ }^{[10]}$ 、惠冰等人 ${ }^{[11]}$ 、宁博等 人 $^{[12]}$ 分别通过数值模拟与室内试验手段研究了动水 渗流环境对渗透注浆扩散范围的影响; Krizek和Per$\mathrm{ez}^{[13]}$ 通过 4 种浆液和 5 种介质进行的 79 组注浆模拟试 验，对不同浆液的稀释特性进行了初步的探索; Zhou 等人 ${ }^{[14]}$ 通过一系列的砂柱注浆室内模型试验，分析了 浆液黏度、砂层孔隙率、注浆流速和压力对水泥基灌 浆材料在多孔介质中扩散的影响; Wang 等人 ${ }^{[15]}$ 设计了 一系列砂土室内模拟试验，通过改变浆液的水灰比和 土体饱和度, 对注浆量和结核体的外形等进行了对比 分析; Bouchelaghem等人 ${ }^{[16]}$ 针对可变形饱和多孔介质 进行了一系列渗透注浆模拟试验，对浆液在扩散过程 中的流体力学性质进行了研究; Zhou等人 ${ }^{[17]}$ 通过模型 试验系统地研究了注浆温度、浆液水灰比、多孔介质 质量分形尺寸对浆液渗透扩散和加固体强度的影响; Axelsson等人 ${ }^{[18]}$ 采用室内砂柱模拟试验，辅以现场试 验，对渗透注浆过程中类水泥浆液止动机制进行了探 讨, 确定了 3 种不同的止动机制.

上述浆液在砂层的扩散研究多集中在水泥浆和普
通的化学浆液方面，由于传统的水泥及黏土类注浆支 护材料颗粒大和可灌性低等特点，其难以注入具有细 微裂隙的介质中; 传统的水玻璃类浆材固结体强度低, 且固结的凝胶产物会对环境产生一定污染 ${ }^{[19,20]}$. 随着 生物和化学等技术的发展，新型注浆新材料，如非水 反应类高聚物 ${ }^{[6]}$ 、水反应高聚物 ${ }^{[21]} 、 \mathrm{CW}$ 环氧树脂 ${ }^{[2]}$ 和微生物菌液 ${ }^{[23]}$ 等实现大批量研制和工程应用, 并取 得了较好的效果. 其中新型非水反应的渗透型高聚物 注浆材料，由于其双组分反应迅速并形成具有一定强 度固结体的优点, 在富水砂层中的注浆应用中具有良 好的发展前景. 然而, 由于其扩散过程中快速固结的 特点, 使得其在富水砂层的扩散规律更为复杂.

为此本文结合现有研究的成果, 制作了一套可视 化的富水砂层渗透注浆模型试验装置，该装置具备可 观察浆液扩散距离、监测注浆前后孔隙水压力变化和 测量堵水率等功能, 以堵水率、渗透系数作为评价富 水砂层渗透注浆堵水防渗效果的性能指标, 开展了以 渗透型高聚物作为注浆浆液的富水砂层渗透注浆堵水 防渗的正交试验，研究了砂层粒径、动水压力、注浆 压力和黏性土含量 4 个因素对注浆堵水防渗效果的影 响, 并分析了影响富水砂层渗透注浆堵水防渗效果的 主控因素以及各单因素对注浆效果的影响特征. 同时, 对模型试验进行了数值模拟, 重点分析了注浆过程中 的浆-水两相渗流场压力、流速和浆液扩散的变化过 程, 并与试验实测浆液扩散距离进行了对比验证, 对新 型渗透型高聚物在富水砂层注浆的工程应用中具有一 定的参考价值.

\section{2 渗透型高聚物在富水砂层中注浆模型 试验}

\section{1 试验装置}

试验装置由注浆装置、富水砂层模拟装置和监测 检测装置3部分构成, 该试验主要是利用在高处悬挂的 水桶通过管路连接砂层模拟装置的进水孔提供恒定水 头压力, 通过高压注浆机提供的注浆压力将浆液从中 心点注入到水平放置的砂层模拟装置中, 试验装置及 示意图如图1和2所示.

\section{1 .1 注浆装置}

本试验注浆装置由双组分聚氨酯注浆机、渗透型 


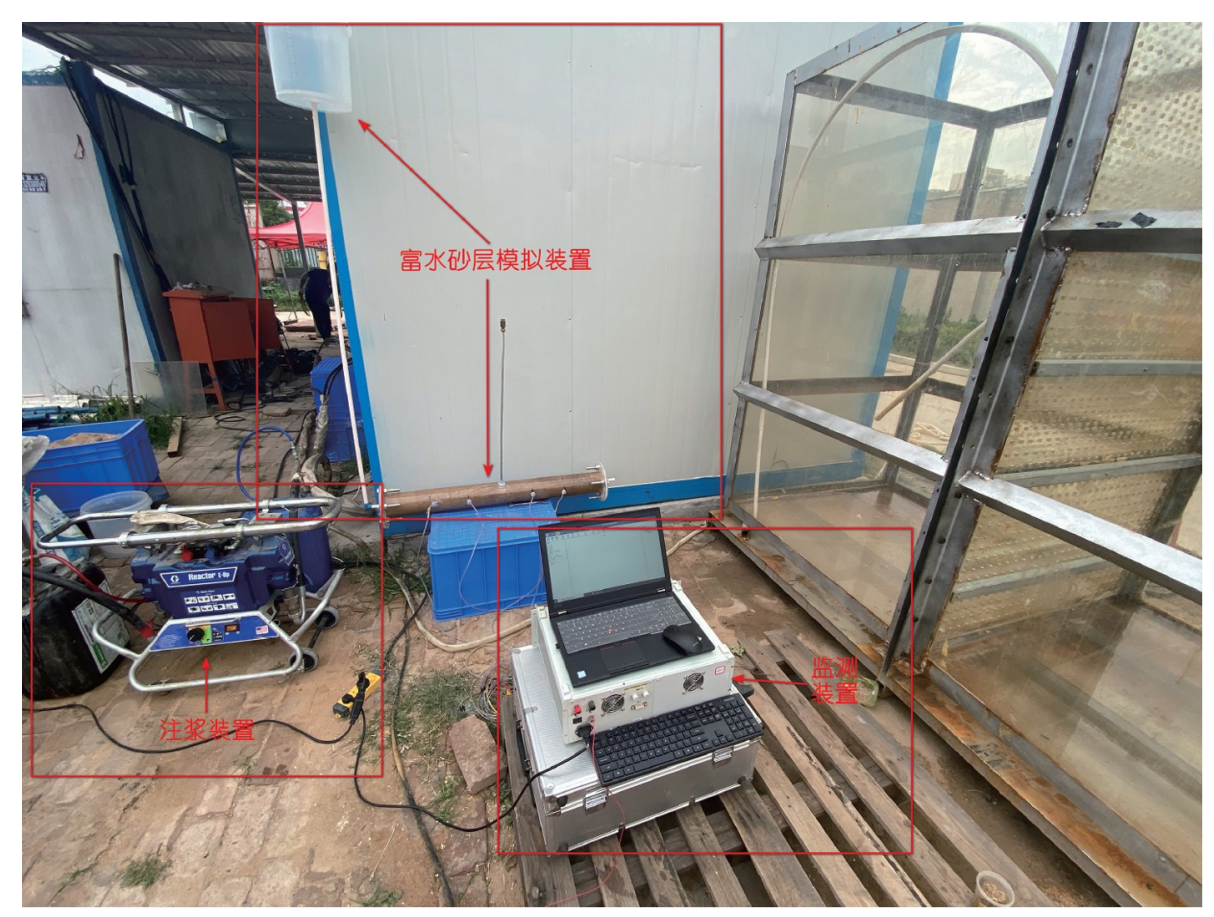

图 1 (网络版彩图)富水砂层注浆试验装置

Figure 1 (Color online) Watery sandy stratum grouting test setup.

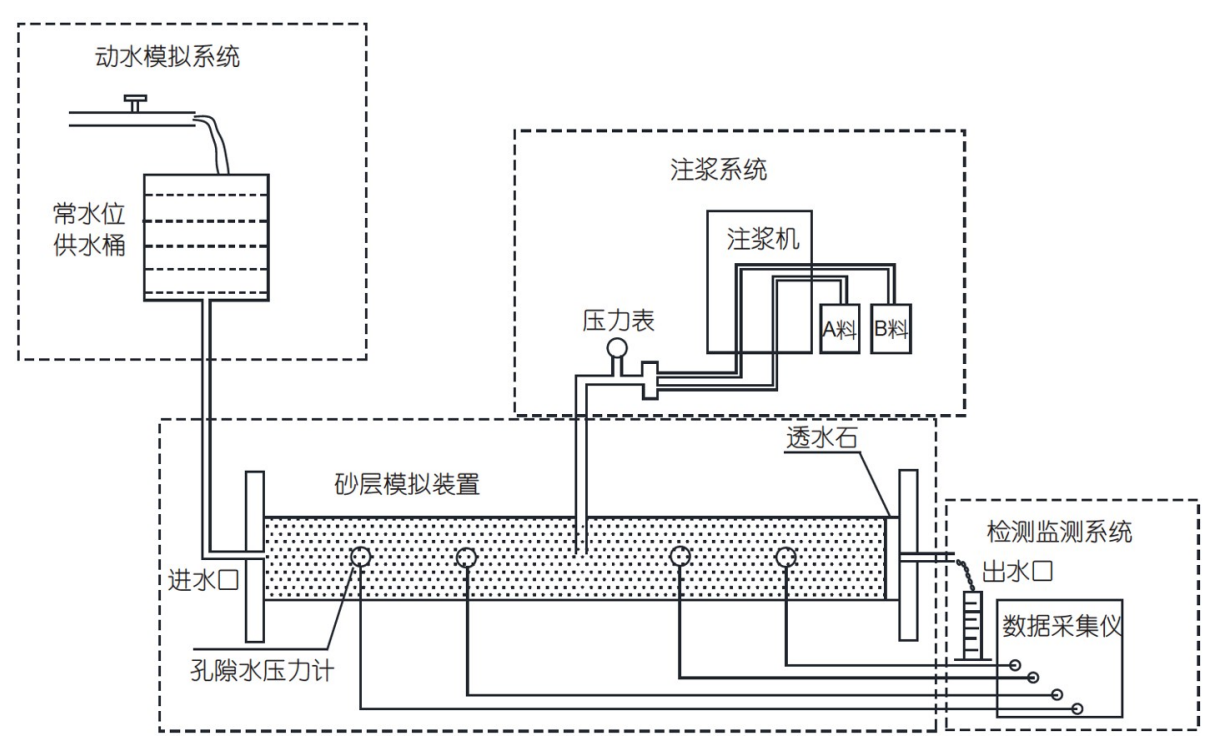

图 2 试验装置示意图

Figure 2 Schematic diagram of the test setup.

聚氨酯浆液桶、高压注浆管和注浆枪组成. 其中该试 验中所采用的注浆机型号为Reactor E-8 p, 其最大注 浆压力可达 $13.8 \mathrm{MPa}$.

\subsection{2富水砂层模拟装置}

本试验的砂层模拟容器采用长度 $1 \mathrm{~m}$ 、内径 $10 \mathrm{~cm}$ 和壁厚 $1 \mathrm{~cm}$ 的透明有机玻璃管，有机玻璃管两端是用 
螺栓紧固的法兰和盲板进行封堵，法兰和盲板之间采 用橡胶垫密封；玻璃管两端的盲板正中间各开一个直 径 $20 \mathrm{~mm}$ 的圆孔作为进水孔和出水孔，出水孔盲板内 侧放置一块直径 $10 \mathrm{~cm}$ 的圆形透水石, 外侧连接与出 水孔同直径、长度为 $5 \mathrm{~cm}$ 的圆管用于排水; 进水孔盲 板内侧粘贴一块超细滤网, 外侧与动水模拟管路连接.

恒定水头压力模拟装置采用满水容量为 $10 \mathrm{~L}$ 的圆 柱形水桶, 水桶由安全绳与在高度 $2.5 \mathrm{~m}$ 处的滑轮进行 支撑悬挂, 悬挂高度可根据试验要求调节, 试验时通过 自来水管向水桶内持续注水，保持桶内的恒定水量为 $5 \mathrm{~L}$ ，水桶底部外连设有堵水开关的坚直管路，管路与 砂层模拟容器的进水孔相连.

\subsection{3 监测与检测装置}

监测与检测装置包括孔隙水压力计、动态信号采 集仪、量筒和渗透仪。试验采用 4 个量程为 $100 \mathrm{kPa}$ 的 DMKY 型孔隙水压力计, 配备动态信号采集仪，信号 采集后可对注浆前后水平放置的模拟砂层中心点两侧 对称的 15 和 $30 \mathrm{~cm}$ 位置处的孔隙水压力变化进行分析, 压力计布置如图3所示; 采用量程为 $1 \mathrm{~L}$ 的量杯测量出 水孔不同时间内的出水量，用来分析注浆堵水的堵水 率和堵水时间; 考虑到注浆后砂层的渗透性较弱, 采 用TST-55型变水头渗透仪测试注浆固结体的渗透系 数, 如图4所示.

\section{2 试验材料}

\subsection{1 浆液材料}

本试验采用的注浆材料是团队自主研发的渗透型 高聚物化学浆液, 以改性聚氨酯为主剂, 辅助了一定的 添加剂，使其在浆液黏度、反应速度调整等方面优于 常用的聚氨酯注浆材料. 试验用的双组分浆液的性能

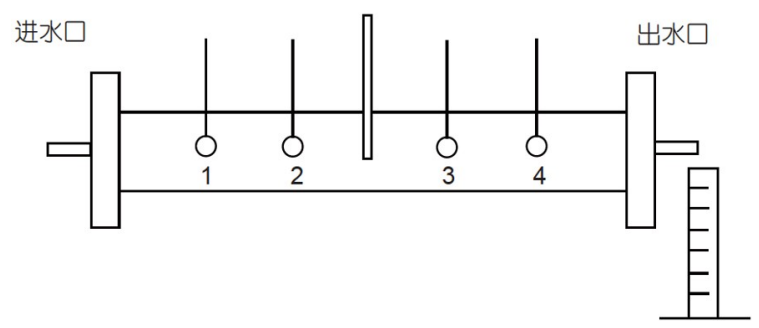

图 3 孔隙水压力计布置示意图

Figure 3 Schematic diagram of pore water pressure meter arrangement.
指标如表1所示, 浆液照片如图5所示.

\subsection{2 砂样}

试验采用的砂为河南地区河砂，根据相关要求 ${ }^{[24]}$ 经过篮孔分别为 $2,1.5,1,0.5$ 和 $0.25 \mathrm{~mm}$ 的篮网笛分

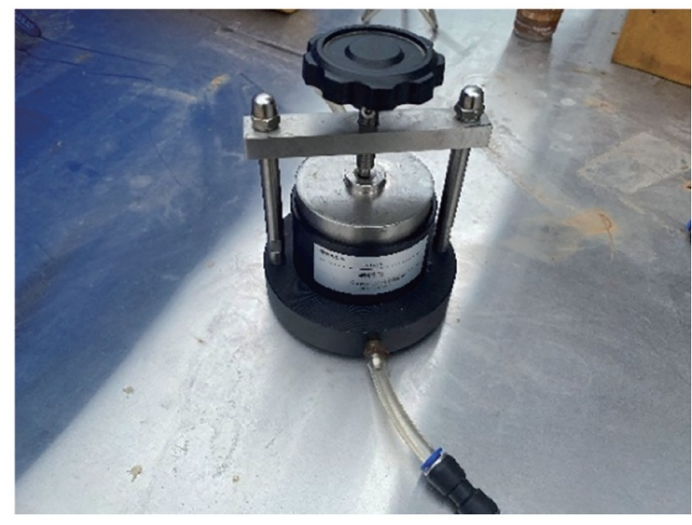

图 4 (网络版彩图)TST-55型渗透仪

Figure 4 (Color online) TST-55 type penetrometer.

表 $1 \mathrm{~A}$ 和B组分浆液性能指标

Table 1 Performance indexes of A and B component slurries

\begin{tabular}{cc}
\hline 项目 & 指标 \\
\hline 浆液黏度 $\left((23 \pm 2){ }^{\circ} \mathrm{C}\right)(\mathrm{mPa} \mathrm{s})$ & $\leq 50$ \\
浆液密度 $\left(\mathrm{g} / \mathrm{cm}^{3}\right)$ & $1.0 \sim 1.2$ \\
固结体拉伸强度 $(\mathrm{MPa})$ & $\geq 1.8$ \\
固结体扯断伸长率 $(\%)$ & $\geq 80$ \\
抗压破坏强度(压缩 $50 \%$ 时) $(\mathrm{MPa})$ & $\geq 20$ \\
反应固结时间 $(\mathrm{min})$ & $\leq 10$ \\
\hline
\end{tabular}

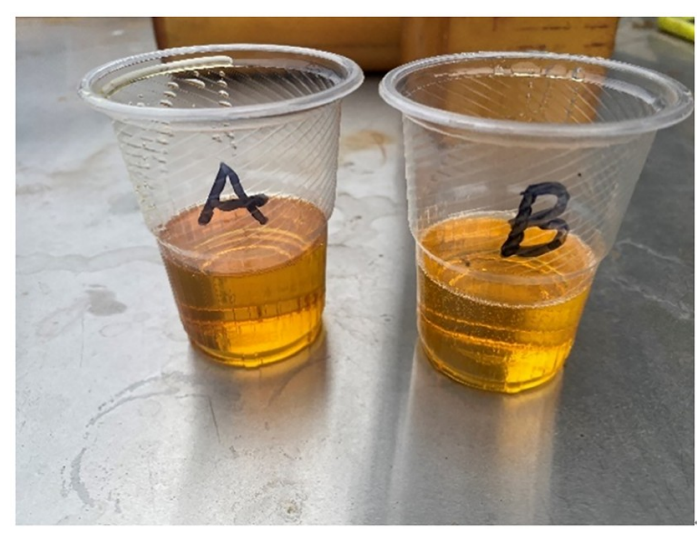

图 5 (网络版彩图)A和B组分浆液

Figure 5 (Color online) A and B component slurry. 
后, 分为不同粒径的砂样, 图6和7分别为振动笁和篎分 后的砂样.

试验不考虑砂土的密实度对注浆效果的影响，为 保证被注砂层的密实程度保持一致，消除因被注砂层 密实程度不同所导致的试验结果差异，试验中采用烘 干的黏性土与砂制作被注砂层试样，将被注砂层的干 密度统一确定为 $1.80 \mathrm{~g} / \mathrm{cm}^{3}$, 在被注砂层试样制作过程 中计量每次装进去砂的重量，根据设定的干密度和装 置尺寸，换算出每次装砂夯填后的填充高度，用夯锤 控制填充高度. 不同粒径和黏性土含量所对应的砂层 试样基本物理指标如表2所示, 根据相关研究 ${ }^{[25]}$, 试验 中每一组分砂样通过定制的纱网篮分, 计算出每组分

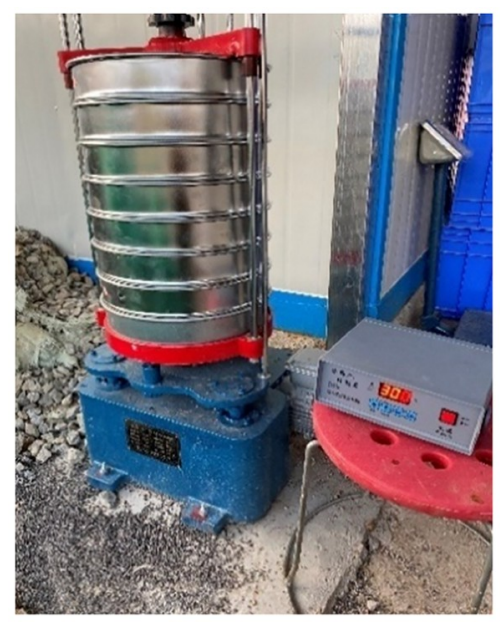

图 6 (网络版彩图)振动笁装置

Figure 6 (Color online) Vibrating sieve device.

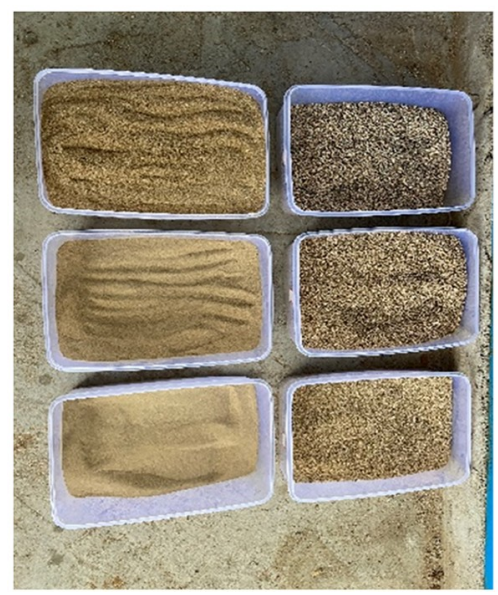

图 7 (网络版彩图)䇛分后砂样

Figure 7 (Color online) Sand sample after sieving.
表 2 被注砂层基本物理指标

Table 2 Basic physical indicators of the injected sand layer

\begin{tabular}{|c|c|c|c|c|}
\hline $\begin{array}{c}\text { 砂层粒径 } \\
(\mathrm{mm})\end{array}$ & $\begin{array}{c}\text { 平均颗粒直径 } \\
D_{\mathrm{P}}(\mathrm{mm})\end{array}$ & $\begin{array}{l}\text { 黏性土 } \\
\text { 含量 }(\%)\end{array}$ & $\begin{array}{l}\text { 干密度 } \\
\left(\mathrm{g} / \mathrm{cm}^{3}\right)\end{array}$ & $\begin{array}{c}\text { 渗透系数 } \\
(\mathrm{cm} / \mathrm{s})\end{array}$ \\
\hline \multirow{4}{*}{$1.5 \sim 2.0$} & \multirow{4}{*}{1.9} & 0 & \multirow{4}{*}{1.80} & $3.324 \times 10^{-2}$ \\
\hline & & 3 & & $8.526 \times 10^{-3}$ \\
\hline & & 6 & & $4.237 \times 10^{-3}$ \\
\hline & & 9 & & $2.144 \times 10^{-3}$ \\
\hline \multirow{4}{*}{$1.0 \sim 1.5$} & \multirow{4}{*}{1.3} & 0 & \multirow{4}{*}{1.80} & $2.912 \times 10^{-2}$ \\
\hline & & 3 & & $7.213 \times 10^{-3}$ \\
\hline & & 6 & & $3.132 \times 10^{-3}$ \\
\hline & & 9 & & $1.114 \times 10^{-3}$ \\
\hline \multirow{4}{*}{$0.5 \sim 1.0$} & \multirow{4}{*}{0.8} & 0 & \multirow{4}{*}{1.80} & $2.437 \times 10^{-2}$ \\
\hline & & 3 & & $4.252 \times 10^{-3}$ \\
\hline & & 6 & & $1.047 \times 10^{-3}$ \\
\hline & & 9 & & $7.563 \times 10^{-4}$ \\
\hline \multirow{4}{*}{$0.25 \sim 0.5$} & \multirow{4}{*}{0.4} & 0 & \multirow{4}{*}{1.80} & $1.921 \times 10^{-2}$ \\
\hline & & 3 & & $2.534 \times 10^{-3}$ \\
\hline & & 6 & & $9.853 \times 10^{-4}$ \\
\hline & & 9 & & $5.334 \times 10^{-4}$ \\
\hline
\end{tabular}

砂样平均颗粒直径.

\section{3 正交试验设计}

本试验注浆的目的是为了提高砂层的堵水抗渗性 能, 根据相关的研究 ${ }^{[10,26]}$, 水泥浆液或普通化学浆液在 富水砂层中扩散时, 动水压力、注浆压力、砂层粒径 和黏性土含量对渗透注浆的堵水效果起到关键作用, 本文试验部分选取以上 4 个因素作为本次渗透注浆堵 水试验的影响因素, 进行 4 因素 $\times 4$ 水平的正交试验, 选 择堵水率和渗透系数作为判断本次注浆试验堵水抗渗 效果的性能指标.

\subsection{1 动水压力}

本试验动水压力是由在高处悬挂的恒定水位为 $5 \mathrm{~L}$ 的水桶提供, 根据试验需要设定 $0.5,1.0,1.5$ 和 $2.0 \mathrm{~m}$ 的 4 个水头高度来提供不同的动水压力.

\subsection{2 注浆压力}

根据高分子材料在砂层渗透注浆中压力的应用, 选定本试验注浆压力的 4 个水平分别为 $200,300,400$ 和 $500 \mathrm{kPa}$. 


\subsection{3 砂层粒径与黏性土含量}

试验中选取被注砂层颗粒粒径的 4 个水平分别为 1.5 2.0, 1.0 1.5, 0.5 1.0, 0.25 0.5 mm; 选取被注砂层 黏性土含量的 4 个水平分别为 $0 \%, 3 \%, 6 \%, 9 \%$, 其具体 砂层特征参数如表 2 所示.

\section{4 试验安排}

本次富水砂层的注浆堵水抗渗试验为 4 因素 $\times 4$ 水 平的正交试验，不考虑因素间的交互作用，正交表采用 $\mathrm{L}_{16}\left(4^{4}\right)$, 正交试验安排如表3所示.

考虑到注浆试验过程受人为操作因素影响较大, 可能会造成试验结果离散性大，为尽量减小试验操作 误差对试验结果的影响, 在试验过程中每组试验均重 复进行 6 次, 去掉明显异常数据, 最终数据取有效数据 的平均值.

\section{5 试验步骤}

(1) 砂层模拟装置出水口一侧放置直径 $10 \mathrm{~cm}$ 透水 石, 安装盲板, 在进水口一侧装入每组试验需要的砂
土, 根据要求控制砂层密实度为 $1.80 \mathrm{~g} / \mathrm{cm}^{3}$, 填满后安 装进水口盲板, 盲板内侧设置一块直径 $10 \mathrm{~cm}$ 超细 滤网;

(2) 在水平放置的砂层模拟装置设定的位置布设 孔隙水压力计; 在砂层模拟装置正中间安设注浆管, 并用热熔胶封堵孔隙, 调试注浆机为正常的工作状态;

（3）保持动水模拟装置水桶恒定水位为 $5 \mathrm{~L}$ ，根据 每组试验的设计使水桶悬挂在不同高度处, 连接管路, 打开供水阀门使模拟砂层被动水流动穿透，待出水口 水流量稳定后, 孔隙水压力计开始读取记录数据, 出 水口下方放置量杯并测量出水口每分钟出水流量;

（4）启动注浆机, 当压力表达到试验要求注浆压力 时, 打开注浆阀门开始注浆, 保持注浆压力不变, 一直 到浆液注不进去为止;

(5) 注浆结束后, 保持动水压力不变, 观察出水口 处水流变化，测量注浆结束 $10 \mathrm{~min}$ 时出水口每分钟的 出水流量; 将注浆后的砂层模拟装置放置于室温为 $26^{\circ} \mathrm{C}$ 的恒温养护室 $1 \mathrm{~d}$;

(6) 对养护 $1 \mathrm{~d}$ 后的砂层模拟装置拆模, 取出注浆

表 3 正交试验安排及试验结果

Table 3 Orthogonal test arrangement and test results

\begin{tabular}{|c|c|c|c|c|c|c|}
\hline \multirow[b]{2}{*}{ 试验编号 } & \multirow{2}{*}{$\begin{array}{c}\text { 因素1 } \\
\text { 动水压力 } \\
\text { (水头高度) }(\mathrm{m})\end{array}$} & \multirow{2}{*}{$\begin{array}{c}\text { 因素2 } \\
\text { 注浆压力 } \\
(\mathrm{kPa})\end{array}$} & \multirow{2}{*}{$\begin{array}{c}\text { 因素3 } \\
\text { 砂层粒径 } \\
(\mathrm{mm})\end{array}$} & \multirow{2}{*}{$\begin{array}{c}\text { 因素4 } \\
\text { 黏性土含量 } \\
(\%)\end{array}$} & \multicolumn{2}{|c|}{ 注浆后测试结果 } \\
\hline & & & & & $\begin{array}{c}\text { 堵水率 } \\
(\%)\end{array}$ & $\begin{array}{c}1 \mathrm{~d} \text { 渗透系数 } \\
(\mathrm{cm} / \mathrm{s})\end{array}$ \\
\hline 1 & 0.5 & 200 & $1.5 \sim 2.0$ & 0 & 93.79 & $5.55 \times 10^{-7}$ \\
\hline 2 & 0.5 & 300 & $1.0 \sim 1.5$ & 3 & 94.32 & $5.75 \times 10^{-7}$ \\
\hline 3 & 0.5 & 400 & $0.5 \sim 1.0$ & 6 & 95.03 & $5.12 \times 10^{-7}$ \\
\hline 4 & 0.5 & 500 & $0.25 \sim 0.5$ & 9 & 95.44 & $5.07 \times 10^{-7}$ \\
\hline 5 & 1.0 & 200 & $1.0 \sim 1.5$ & 6 & 93.13 & $6.97 \times 10^{-7}$ \\
\hline 6 & 1.0 & 300 & $1.5 \sim 2.0$ & 9 & 95.24 & $7.01 \times 10^{-7}$ \\
\hline 7 & 1.0 & 400 & $0.25 \sim 0.5$ & 0 & 94.11 & $4.01 \times 10^{-7}$ \\
\hline 8 & 1.0 & 500 & $0.5 \sim 1.0$ & 3 & 96.27 & $3.94 \times 10^{-7}$ \\
\hline 9 & 1.5 & 200 & $0.5 \sim 1.0$ & 9 & 92.86 & $8.04 \times 10^{-7}$ \\
\hline 10 & 1.5 & 300 & $0.25 \sim 0.5$ & 6 & 93.06 & $6.33 \times 10^{-7}$ \\
\hline 11 & 1.5 & 400 & $1.5 \sim 2.0$ & 3 & 95.87 & $4.57 \times 10^{-7}$ \\
\hline 12 & 1.5 & 500 & $1.0 \sim 1.5$ & 0 & 97.02 & $3.54 \times 10^{-7}$ \\
\hline 13 & 2.0 & 200 & $0.25 \sim 0.5$ & 3 & 92.75 & $6.21 \times 10^{-7}$ \\
\hline 14 & 2.0 & 300 & $0.5 \sim 1.0$ & 0 & 93.87 & $4.89 \times 10^{-7}$ \\
\hline 15 & 2.0 & 400 & $1.0 \sim 1.5$ & 9 & 96.92 & $5.88 \times 10^{-7}$ \\
\hline 16 & 2.0 & 500 & $1.5 \sim 2.0$ & 6 & 97.52 & $4.45 \times 10^{-7}$ \\
\hline
\end{tabular}


后的固结体, 取样进行渗透系数测试.

\section{3 模型试验的数值模拟}

COMSOL Multiphysics软件主要以有限元法为基 础, 通过求解偏微分方程(组)来实现真实物理现象的 模拟仿真，本节内容采用该软件多孔介质和地下水流 的两相达西定律接口，重点模拟分析了模型试验注浆 过程中渗流场压力、流速和浆液扩散范围的变化.

\section{1 控制方程}

采用的物理模型认为砂层为各向同性的不可压缩 介质, 水和浆液均为不可压缩的各向同性的流体, 采用 的控制方程如下.

浆液质量守恒方程:

$$
-\left[\frac{\partial\left(\rho_{\mathrm{o}} v_{\mathrm{o} x}\right)}{\partial_{x}}+\frac{\partial\left(\rho_{\mathrm{o}} v_{\mathrm{o} y}\right)}{\partial_{y}}+\frac{\partial\left(\rho_{\mathrm{o}} v_{\mathrm{o} z}\right)}{\partial_{z}}\right]=\frac{\partial\left(\varphi \rho_{\mathrm{o}} S_{\mathrm{o}}\right)}{\partial_{t}} .
$$

水的质量守恒方程:

$$
-\left[\frac{\partial\left(\rho_{\mathrm{w}} v_{\mathrm{w} x}\right)}{\partial_{x}}+\frac{\partial\left(\rho_{\mathrm{w}} v_{\mathrm{w} y}\right)}{\partial_{y}}+\frac{\partial\left(\rho_{\mathrm{w}} v_{\mathrm{w} z}\right)}{\partial_{z}}\right]=\frac{\partial\left(\varphi \rho_{\mathrm{w}} S_{\mathrm{w}}\right)}{\partial_{t}} .
$$

动力学方程运用广义达西定律:

$$
v=\frac{K}{\mu} \nabla p \text {. }
$$

控制方程:

$$
S_{\mathrm{o}}+S_{\mathrm{w}}=1 \text {, }
$$

式中, $\rho_{\mathrm{o}}$ 是浆液的密度, $\rho_{\mathrm{w}}$ 是水的密度, $\varphi$ 是砂层的孔隙 率, $S_{0}$ 是浆液相的饱和度, $S_{\mathrm{w}}$ 是水相的饱和度, $v$ 是渗流 场的速度， $\mu$ 是水和浆液黏度加权平均后的流体黏度， $K$ 是砂层介质的渗透率, $p$ 是渗流场的压力.

\section{2 有限元模型}

由于采用的物理模型认为砂层为各向同性的不可 压缩介质, 通过对注浆模拟过程的简化, 进行了试验装 置俯视面的二维模型注浆过程的数值分析，建立的模 型尺寸为长 $1 \mathrm{~m}$ 、高 $0.1 \mathrm{~m}$ 的长方形，注浆孔位于模型 的中心, 注浆孔直径为 $1 \mathrm{~cm}$. 模型的边界设定为: 左边 界设为压力进水边界, 右边界设为流出边界, 上下边界 为无流动的边界, 注浆孔处设定为压力进浆液边界. 模 型及网格划分如图8所示.

\section{4 试验结果与数值模拟结果分析}

\section{1 模型试验结果}

富水砂层注浆的固结体试样脱模后如图9所示, 在 不同工况下的试验, 浆液扩散距离不一样, 形成的注浆 固结体长短也不一样, 但注浆固结体表观质量较好, 在 固结有效范围内不存在明显的薄弱区，验证了注浆装 置的可用性和注浆浆液的有效性.

富水砂层注浆后的堵水效果可用堵水率来表示, 注浆后的渗流量理论上应小于注浆前的渗流量，渗流 量减小的值与原渗流量值的比值的百分数定义为堵水 率，通过注浆前后渗流量变化，换算成堵水率，可以更 加直观地评价注浆堵水的效果．堵水率计算公式如下 式所示:

$S E(\%)=\frac{Q_{\mathrm{o}}-Q_{\mathrm{g}}}{Q_{\mathrm{o}}} \times 100 \%$,

式中, $S E$ 为堵水率 $(\%), Q_{0}$ 为注浆前渗流量 $(\mathrm{mL} / \mathrm{min})$, $Q_{\mathrm{g}}$ 为注浆后渗流量 $(\mathrm{mL} / \mathrm{min})$.

渗透系数可以表征砂层注浆固结体的防渗性能, 注浆固结体的渗透系数越小说明砂层注浆固结体的防 渗能力越强. 由于被注浆后的砂层渗透系数较小, 本次 试验渗透系数的检测采用TST-55型渗透仪进行变水头

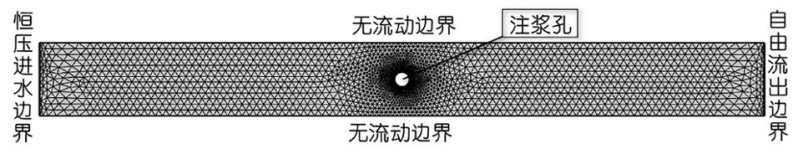

图 8 几何模型与网格划分

Figure 8 Geometric model and meshing.

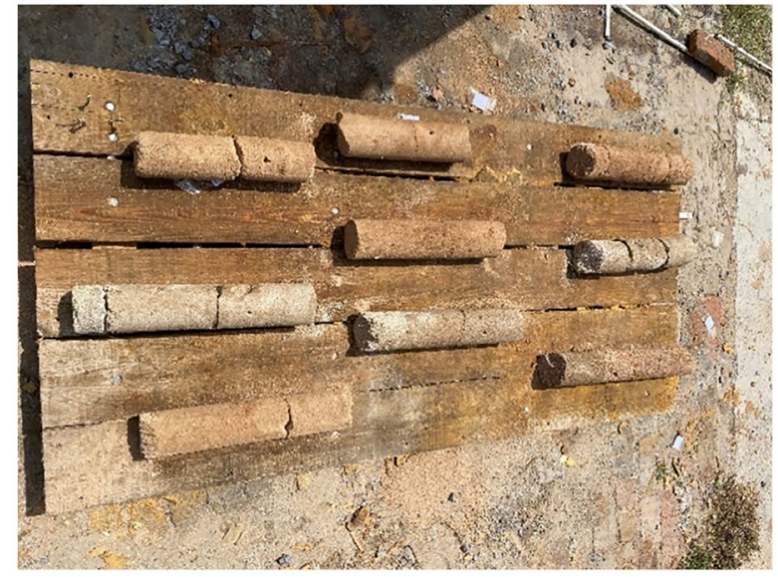

图 9 (网络版彩图)脱模后注浆固结体

Figure 9 (Color online) Grout consolidation after demoulding. 
试验, 如图10所示. 由表 3 可知在注浆后的 $10 \mathrm{~min}$ 内, 堵 水率可达 $92.75 \%$ 97.52\%不等，注浆 $1 \mathrm{~d}$ 后的固结体渗 透系数可达到 $10^{-7} \mathrm{~cm} / \mathrm{s}$ 的数量级, 得到了非常好的堵 水防渗效果.

\section{2 注浆堵水效果主控因素分析}

采用极差分析方法，分析影响富水砂层注浆堵水 效果的主控因素, 极差分析的结果如表4所示.

表4中的 $K_{1}, K_{2}, K_{3}, K_{4}$ 分别为各个因素在 4 个水平 下的堵水率平均值，反映各因素不同水平对注浆堵水 率的影响, $R$ 为某一因素不同水平下堵水率的极差, 用 来反映这一因素的水平变化对于注浆堵水率影响程度 的大小, 极差大代表该因素为影响堵水率的主要因素. 由表4可知, $R_{2}>R_{3}>R_{1}>R_{4}$, 上述 4 个因素对砂层注浆后 堵水效果(堵水率)的影响次序为: 注浆压力 $>$ 砂层粒径 $>$ 动水压力>黏性土含量，即注浆压力是影响堵水效果 的主控因素.

单因素影响曲线是对正交试验极差分析结果的形 象描绘，把表4中 4 个因素在各个水平下的结果均值绘 制成影响曲线, 如图11所示.

从图11(a)可以看出，堵水率与水头高度之间没有 明显的线性关系，在 $0.5 \sim 1.5 \mathrm{~m}$ 的水头高度范围内，堵 水率随水头高度增加而缓慢增长，在1.5 2.0 $\mathrm{m}$ 的水头 高度范围内, 堵水率增长速率较大. 分析原因是水在 砂层模拟装置中的扩散需要克服砂粒的摩擦阻力, $0.5 \sim 1.5 \mathrm{~m}$ 的水头高度范围内提供的动水压力使得水 在扩散的过程中受摩擦阻力影响较大, 出水口渗流速 率随水头高度增加缓慢增长; 当水头高度大于 $1.5 \mathrm{~m}$ 后 提供的动水压力使得水在扩散过程中受摩擦阻力的影 响较小, 出水口的渗流速率增大得较快. 其次, 在注浆

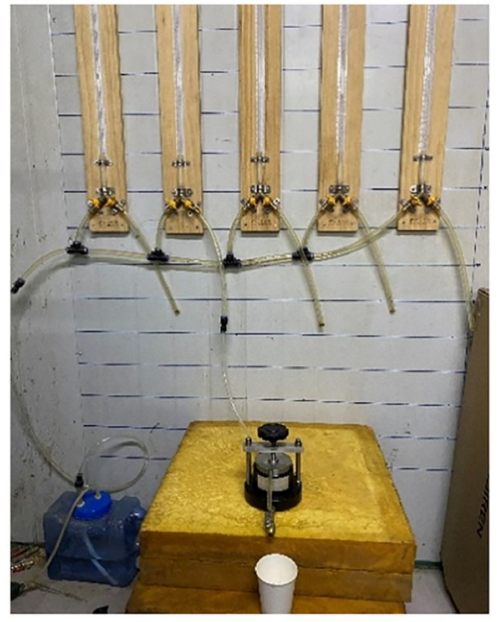

图 10 (网络版彩图)变水头渗透试验

Figure 10 (Color online) Variable head penetration test.

后因为渗透型高聚物浆液良好的性能, 水头高度在 $2 \mathrm{~m}$ 范围内仍起到较好的堵水效果, 注浆后出水口的渗流 速率很小, 就得到了图11(a)中堵水率随水头高度变化 的增长趋势.

从图11(b)可以看出，注浆压力与注浆堵水率有明 显的线性正相关的趋势关系, 堵水率随注浆压力增加 而增大，分析原因是注浆压力越大，浆液在砂层颗粒 孔隙中填充的越充分，扩散的距离越远，所以注浆堵 水的效果越好. 在工程实际中, 若想得到较好的堵水 效果，可以考虑在砂层渗透注浆的压力范围内增大注 浆的压力.

从图11(c)可以看出, 砂层粒径与注浆堵水率也有 线性正相关的趋势关系，堵水率随砂层粒径增大而增 大，但在1.0 1.5 mm之后，堵水率增大有减缓的趋势， 分析原因是砂层粒径值较小时, 受注砂层的孔隙度也

表 4 堵水率极差分析结果 ${ }^{a)}$

Table 4 Results of extreme difference analysis of water plugging rate

\begin{tabular}{ccccc}
\hline 分析指标 & 因素1(动水压力) & 因素2(注浆压力) & 因素3(砂层粒径) & 因素4(䇿性土含量) \\
\hline$K_{1}$ & 94.65 & 93.13 & 95.61 & 94.70 \\
$K_{2}$ & 94.69 & 94.12 & 95.35 & 94.80 \\
$K_{3}$ & 94.70 & 95.48 & 94.51 & 94.69 \\
$K_{4}$ & 95.27 & 96.56 & 93.84 & 95.12 \\
极差 $R_{j}$ & 0.62 & 3.43 & 1.10 & 0.12 \\
因素主 $\rightarrow$ 次 & & 注浆压力>砂层粒径>动水压力>黏性土含量 & \\
\hline
\end{tabular}

a) $K_{j}$ 为水平号为 $j$ 时, 所对应试验结果的平均值, $R_{j}$ 为因素 $j$ 的极差, $j=1,2,3,4$, 下同 

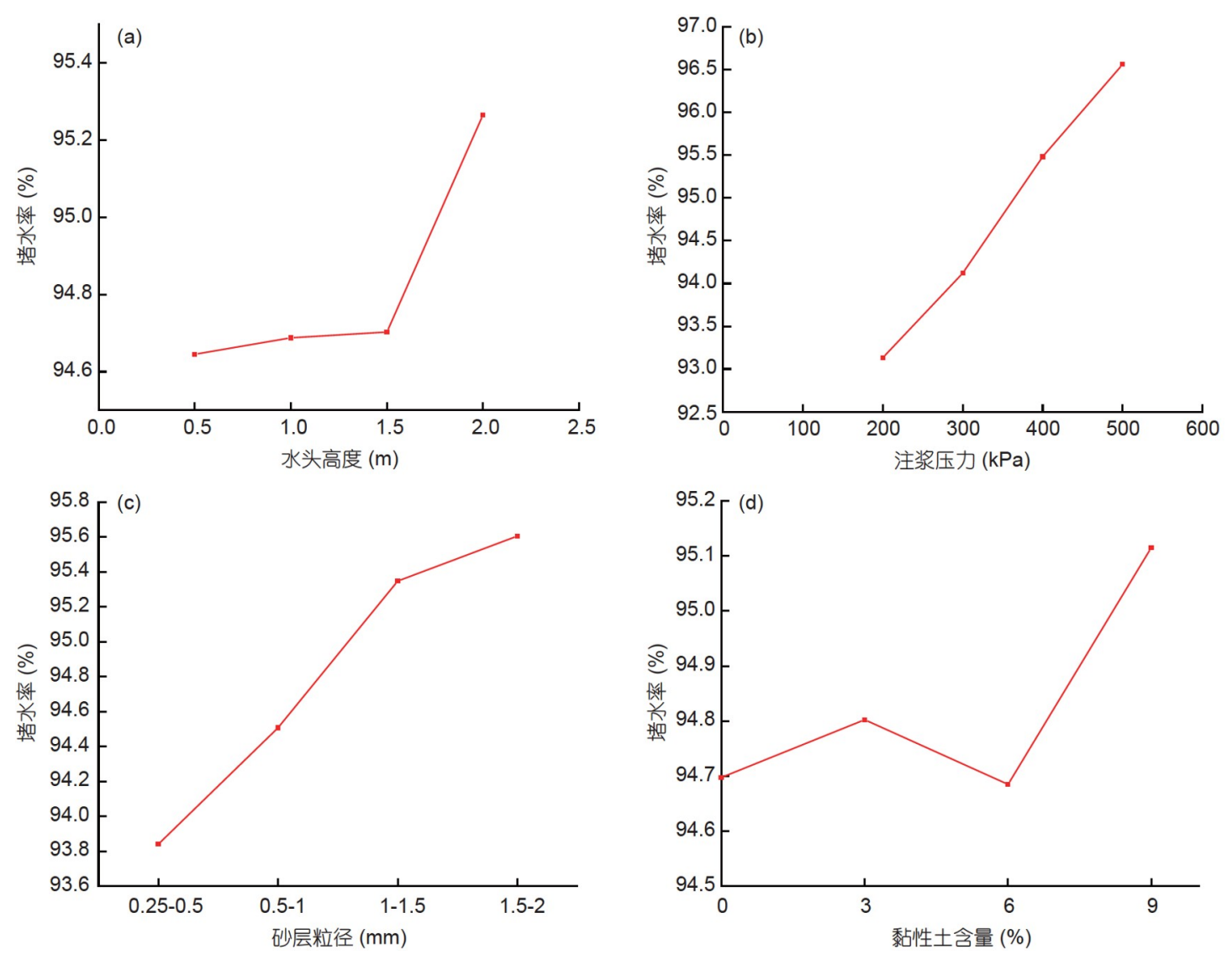

图 11 (网络版彩图)各因素对注浆堵水效果的影响趋势

Figure 11 (Color online) Trend of the influence of various factors on the effect of grouting and water plugging.

较小, 此时高聚物浆液注入性相对来说较低; 随着砂层 粒径值变大, 受注砂层孔隙度也会变大, 高聚物浆液的 注入性随之提高，此阶段高聚物浆液的封堵效果越来 越好；一旦砂层粒径值超过某一特定值，受注砂层孔 隙度进一步增大，此时在水流速度的影响下，在相同 注浆量条件下，高聚物浆液在一定程度上被稀释，封 堵效果在一定程度上会变差.

从图11(d)可以看出黏性土含量与注浆后的堵水 效果关系较为复杂，无明显的线性关系，分析原因是: 根据表2中测得数据, 黏粒含量与砂层注浆前的渗透系 数呈负相关的关系, 黏粒含量越高, 对砂粒间孔隙填充 度越高， $6 \% \sim 9 \%$ 含量的黏粒就能使砂层初始渗透系数 达到 $10^{-4} \mathrm{~cm} / \mathrm{s}$ 数量级. 在黏粒含量 $0 \% \sim 3 \%$ 的范围内, 黏粒对浆液在孔隙中扩散效果产生的负面作用要小于 其对水渗流量变化的影响，浆液凝固后出水口渗流量 的减小梯度要大于注浆前砂层在 $0 \% \sim 3 \%$ 黏粒含量范
围内减小的梯度，堵水率有升高现象; 在 $3 \% \sim 6 \%$ 的范 围内，黏粒含量对浆液在孔隙中扩散效果产生的负面 作用要大于其对水渗流量变化的影响，堵水率有下降 现象; 在黏粒含量 $6 \%$ \% $\%$ 的范围内, 由于砂层初始渗 透系数已达 $10^{-4} \mathrm{~cm} / \mathrm{s}$ 数量级, 注浆前出水口渗流量很 小, 注浆后渗流量随黏粒含量减小的梯度更大, 浆液 扩散后得到的堵水率更大.

\section{3 注浆后抗渗性能主控因素分析}

分析表 3 中注浆后砂层 $1 \mathrm{~d}$ 渗透系数可知, 注浆固 结体 $1 \mathrm{~d}$ 的渗透系数已达到 $10^{-7} \mathrm{~cm} / \mathrm{s}$ 数量级, 未注浆前 砂层的渗透系数在 $10^{-4} \sim 10^{-2} \mathrm{~cm} / \mathrm{s}$ 之间, 表明砂层注浆 后的防渗性能已得到了极大的提高, 能满足工程中绝 大多数的防渗要求. 采用极差分析的方法, 分析影响 富水砂层注浆固结体防渗性能的主控因素, 极差分析 的结果如表5所示. 
由表 5 可知, $R_{2}>R_{4}>R_{1}>R_{3}$, 上述 4 个因素对砂层注 浆后防渗性能的影响次序为: 注浆压力 $>$ 黏性土含量 $>$ 动水压力 $>$ 砂层粒径，即注浆压力是影响防渗性能的 主控因素。把表 5 中 4 个因素在各个水平下的结果均值 绘制影响曲线, 如图12所示.

从图12(a)可以看出, 水头高度对固结体渗透系数 的影响有先增大后减小的趋势，分析原因是在
0.5 1.5 m的水头高度范围内，水和浆液的扩散均受砂 粒摩擦阻力的影响较大，浆液扩散均匀程度受到了一 定的影响, 导致在此水头高度范围内渗透系数有上升 的趋势; 但在1.5 2.0 $\mathrm{m}$ 的水头高度范围内, 由于水扩 散受砂粒摩擦阻力的影响较小，带动浆液在砂粒之间 的扩散, 浆液扩散相对均匀, 固结体的渗透系数明显 降低.

表 5 渗透系数极差分析结果

Table 5 Results of extreme difference analysis of permeability coefficient

\begin{tabular}{ccccc}
\hline 分析指标 & 因素1(动水压力) & 因素2(注浆压力) & 因素3(砂层粒径) & 因素4(黏性土含量) \\
\hline$K_{1}$ & 5.37 & 6.69 & 5.40 & 4.50 \\
$K_{2}$ & 5.48 & 6.00 & 5.54 & 5.12 \\
$K_{3}$ & 5.62 & 4.90 & 5.50 & 5.72 \\
$K_{4}$ & 5.36 & 4.25 & 5.41 & 6.50 \\
极差 $R_{j}$ & 0.26 & 2.44 & 0.14 & 1.22 \\
因素主 $\rightarrow$ 次 & & 注浆压力>黏性土含量>动水压力>砂层粒径 & \\
\hline
\end{tabular}
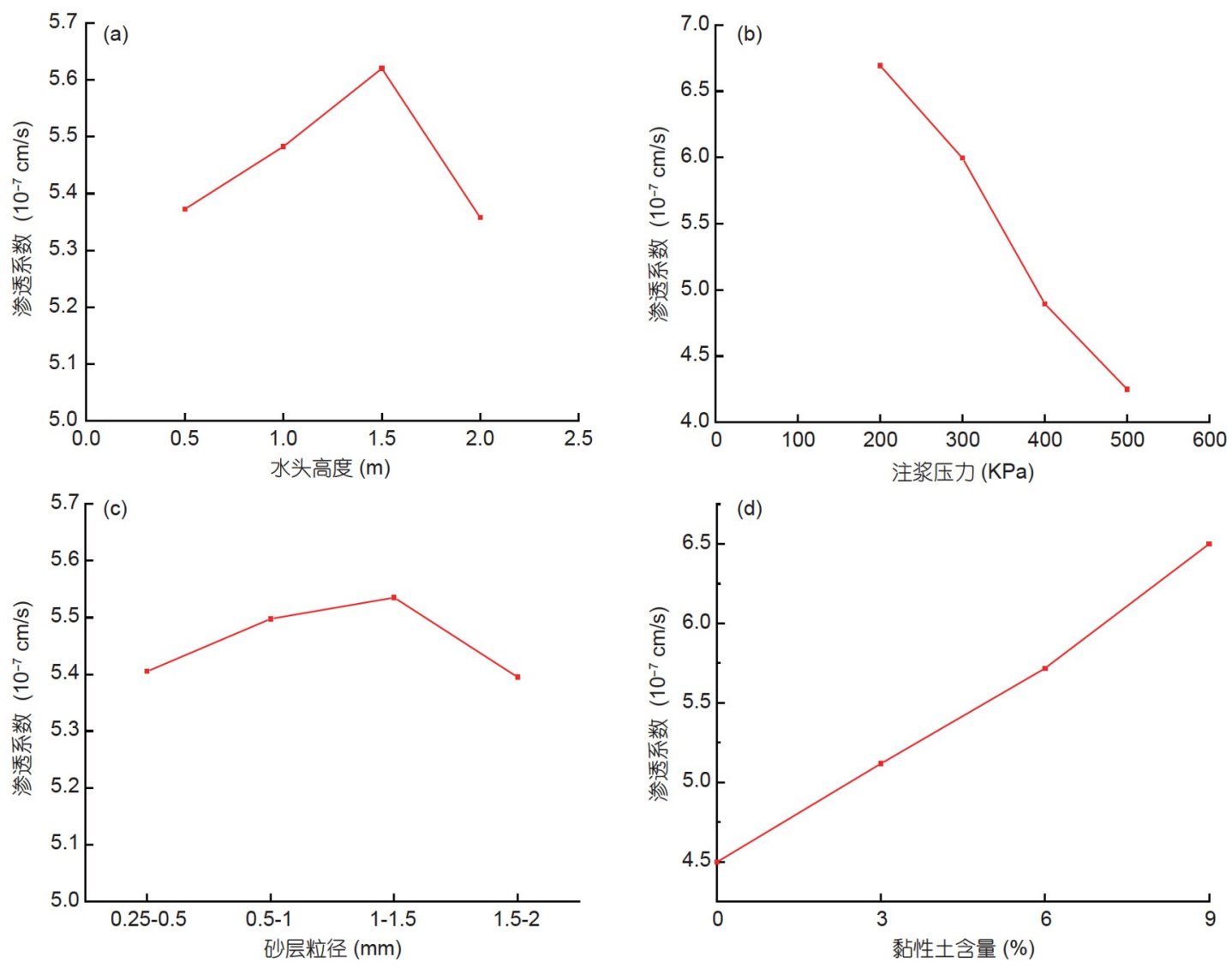

图 12 (网络版彩图)各因素对注浆防渗效果的影响趋势

Figure 12 (Color online) Influence trend of various factors on grouting anti-seepage effect. 
从图12(b)可以看出, 注浆压力与固结体渗透系数 有较为明显的负相关线性关系, 也就是注浆压力与注 浆固结体的防渗性能呈正相关. 注浆压力越大, 浆液 在砂粒孔隙中注入得越充分, 对孔隙中水的排挤效果 越好, 加之双组分浆液反应速度较快, 反应成型后能 较好地填充砂粒之间的孔隙, 使得注浆固结体的渗透 系数显著降低, 大大提升注浆后砂层的防渗性能.

从图12(c)可以看出, 砂层粒径的变化与渗透系数 虽有先增大后减小的趋势, 但这几种粒径下的注浆后 固结体渗透系数在 $5.4 \times 10^{-7} \sim 5.6 \times 10^{-7} \mathrm{~cm} / \mathrm{s}$ 之间, 数值 差距极小, 说明在 $0.25 \sim 2 \mathrm{~mm}$ 范围内砂层粒径的变化 对渗透型高聚物在其中扩散的影响不大.

从图12(d)可以看出, 黏性土含量与砂层注浆固结 体的渗透系数呈正相关的关系, 而由表 3 可知, 注浆前 黏性土含量与砂层的渗透系数呈显著的负相关关系, 黏性土的含量越高, 砂层的初始渗透系数越小. 分析产 生注浆前后这种差别的原因是: 由于黏性土颗粒填充 于沙粒的孔隙之间, 减小了砂层的孔隙率, 从而降低 了原始砂层的渗透系数, 但砂粒之间的黏性土颗粒会 加大浆液注入的难度, 黏性土含量越高, 会使浆液注 入的越少, 对注浆固结体渗透系数的降低具有负面的 作用, 最后造成了黏性土含量与砂层注浆固结体的渗 透系数呈正相关关系.

综上, 从砂层注浆后的堵水效果、防渗性能这两 个方面来看, 注浆压力是控制渗透型高聚物在富水砂 层注浆堵水防渗效果的主控因素. 在渗透注浆允许压 力的一定范围内, 随着注浆压力的增加, 富水砂层注 浆的堵水效果和防渗性能均能显著的提高.

\section{4 注浆过程中孔隙水压力变化监测}

本节内容以设计的第一组试验为例, 分析注浆过 程中对孔隙水压力变化的监测数据, 数据采集仪采集 到注浆前后共 $10 \mathrm{~min}$ 左右的孔隙水压力数据, 如图 13 所示.

由图13可知, 注浆从数据采集的 $35 \mathrm{~s}$ 开始, 注浆开 始前, 砂层各点处孔隙水压力为 $5 \mathrm{kPa}$ 左右. 注浆开始 后, 浆液瞬间被注进砂层中, 对砂层中的水有排挤作 用, 导致压力计 2 和压力计 3 处的孔隙水压力迅速增大; 由于压力计4距离注浆管较远, 距离出水口较近, 压力 消散得较快, 故压力值没有压力计 2 和 3 处的大且压力 变化有延迟; 压力计 1 位于上游的逆水段且距离注浆

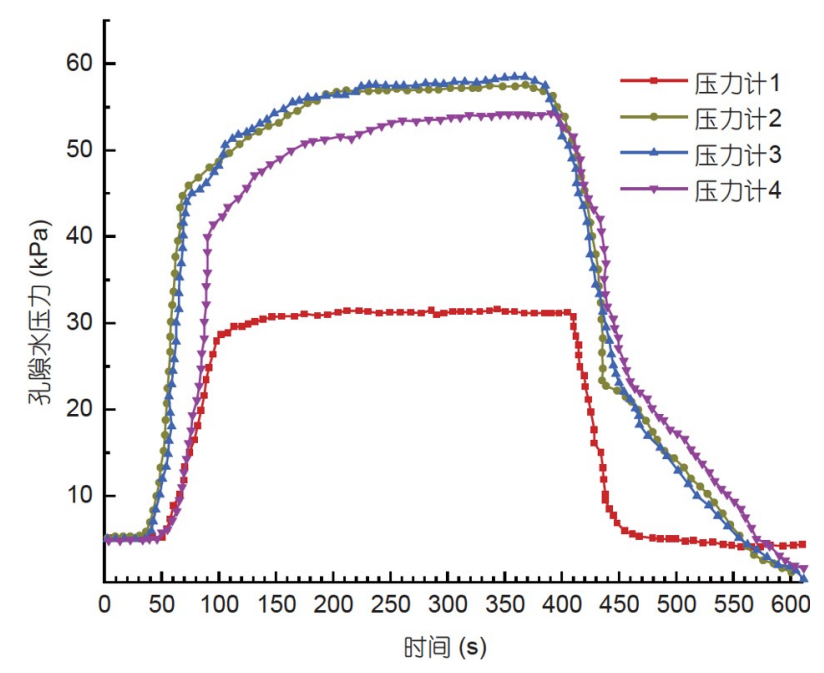

图 13 孔隙水压力随时间变化曲线

Figure 13 Pore water pressure variation curve with time.

管较远, 受浆液排挤的影响有限, 故压力值没有压力 计2 4处的大. 随着注浆的进行, 双组分浆液开始反应, 各压力计处的孔隙水压力持续增大, 但增速放缓. 注浆 进行到 $200 \mathrm{~s}$ 左右时, 压力计 2 和 3 处的压力值达到最大 值, 说明此刻开始浆液反应最为激烈, 随着反应进行, 压力计各处的值维持一段平衡, 基本无变化. $400 \mathrm{~s}$ 左 右时各处的压力开始下降, 说明反应已经大部分完成, $420 \mathrm{~s}$ 时停止注浆, 各处的压力迅速下降. 到600 s左右 时压力计 2 4这三处的孔隙水压力接近为 0 , 此时反 应已基本完成, 由于浆液未扩散到压力计 1 处位置, 反应结束后该处的孔隙水压力和注浆前的压力值 相近.

\section{5 数值模拟结果分析}

以正交试验设计的第 13 组试验参数为例 (水头高 度 $2 \mathrm{~m}$ 、注浆压力 $200 \mathrm{kPa}$ 、砂层粒径 $0.25 \sim 0.5 \mathrm{~mm}$ 、 黏性土含量 $3 \%$ ), 进行水、浆液两相渗流模拟.

\subsection{1富水砂层注浆前渗流压力场模拟}

模拟结果如图14所示, 富水砂层注浆前的渗流场 压力在砂层中从进水边界向出水边界均匀递减, 在出 水边界附近, 渗流场压力接近为零.

\subsection{2 富水砂层注浆后渗流压力场模拟}

模拟结果如图15所示, 由于砂层颗粒对浆液的摩 


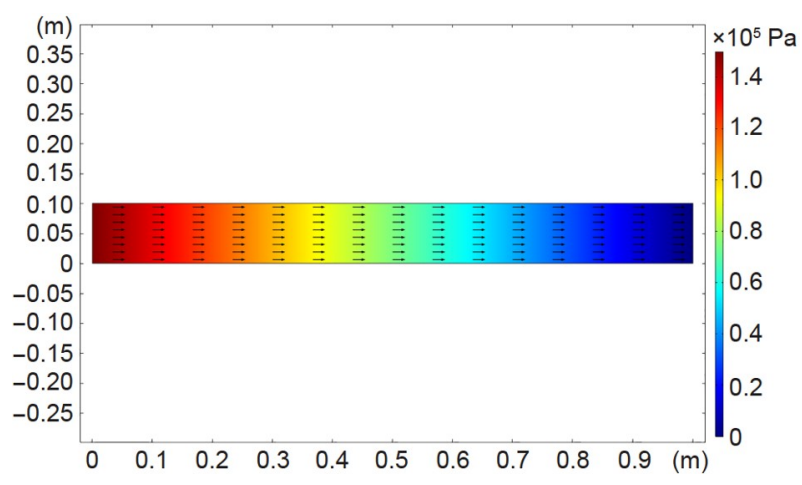

图 14 富水砂层注浆前渗流压力场分布

Figure 14 Distribution of seepage pressure field before grouting in water-rich sand layer.

表面: 压力场 $(\mathrm{Pa})$

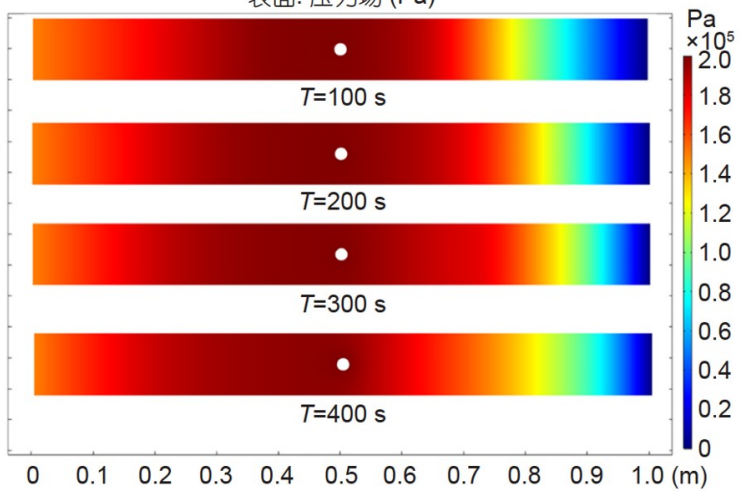

图 15 不同时刻浆-水两相渗流压力场分布

Figure 15 Distribution of pressure field of slurry-water two-phase seepage at different moments.

擦阻力对注浆压力有消散作用，浆液从注浆孔扩散出 去时，顺水段的浆-水渗流场压力整体表现为从注浆孔 向出水边界递减. 在100 300 s内, 随着时间的增加, 出 水边界附近的渗流场压力在浆液持续扩散下逐渐增 大，到400 s时出水边界附近渗流场压力又有所回减， 说明浆液已经扩散到最大距离. 逆水段的浆-水渗流场 压力因为水流与浆液扩散的冲抵作用，变化幅度没有 顺水段大.

\subsection{3 富水砂层注浆后渗流速度场模拟}

浆-水两相渗流速度场模拟结果如图16所示，在注 浆孔附近局部放大，结果显示顺水段渗流的速度在注 浆压力和水流冲刷助力作用下要明显比逆水段的速度 大, 逆水段方向的速度小且方向向左, 这是由于注浆孔
附近浆液在注浆压力作用下有逆水扩散的现象.

\subsection{4 富水砂层注浆后浆-水饱和度模拟}

浆-水饱和度的模拟结果如图17所示, 可以看出在 注浆孔附近浆液的饱和度最高, 距注浆孔越远浆液的 浓度越低，左侧的逆水段存在浆液逆水扩散的现象， 由于水流的冲刷作用，逆水扩散的距离小于顺水扩散 段. $100 \mathrm{~s}$ 时浆液在注浆孔附近扩散形态受水流影响呈 粗圆形，随着时间的增加，100 400 s内浆液逆水段扩 散距离均逐渐增加，而顺水段扩散距离增长速度受水 流冲刷作用要明显大于逆水段扩散距离的增长速度, 400 450 s 浆液由于反应逐渐凝固和砂粒摩擦阻力的 作用, 顺水、逆水段的扩散基本停止.

图18所示的曲线是由浆-水两相饱和度分布云图 提取的 $X-X$ 截面上不同时刻浆液在不同位置处的饱和

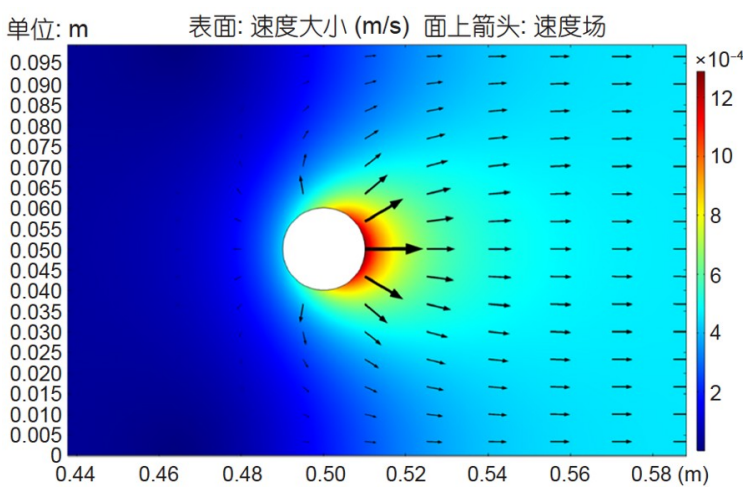

图 16 浆-水两相渗流速度场分布

Figure 16 Velocity field distribution of slurry-water two-phase seepage.

表面: 浆液饱和度

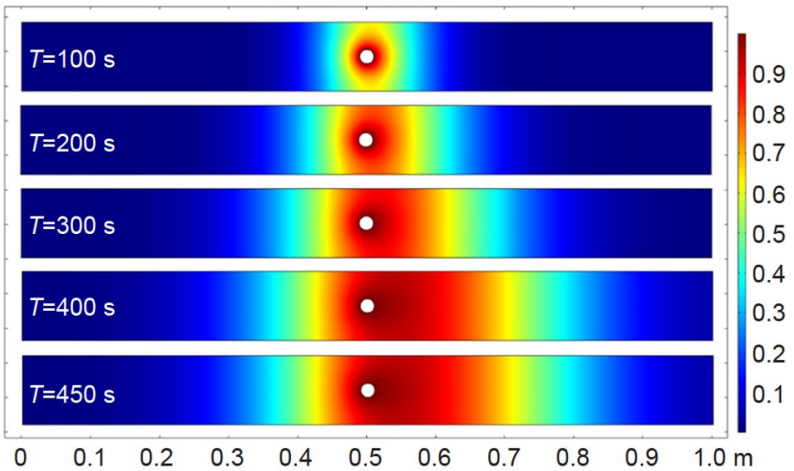

图 17 不同时刻浆-水两相饱和度分布

Figure 17 Distribution of two-phase saturation of pulp-water at different moments. 


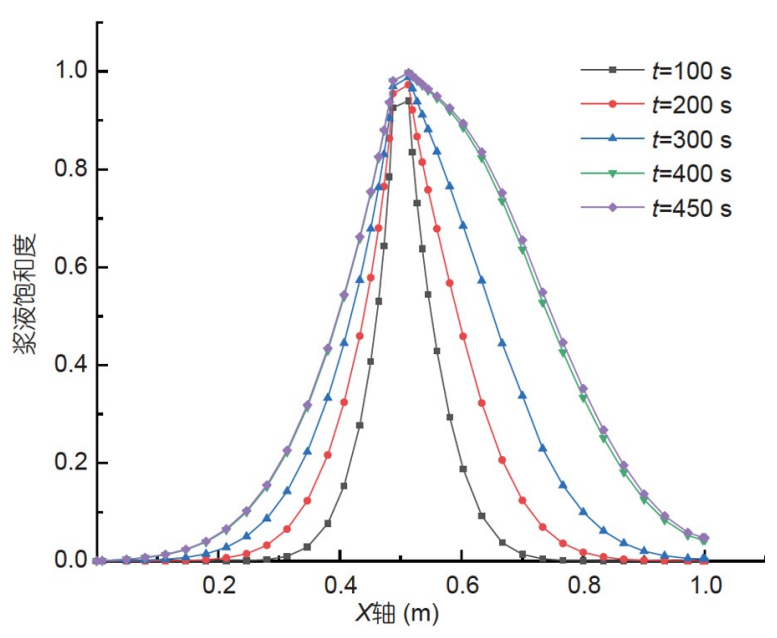

图 18 浆液饱和度随扩散距离变化分布

Figure 18 Distribution of slurry saturation with diffusion distance.

度数据, 更能清楚地展示浆液在顺水、逆水段的扩散 情况. $X=0.5 \mathrm{~m}$ 为注浆孔中心点所在位置, 以饱和度大 于 0.5 的位置代表浆液扩散的有效距离，由图可以得出 浆液在逆水段的扩散距离为 $11 \mathrm{~cm}$ 、顺水段的扩散距 离为 $26 \mathrm{~cm}$. 正交试验设计的第 13 组试验注浆后固结体 脱模后如图 19 所示，实测浆液逆水段扩散距离为 $13 \mathrm{~cm}$ 、顺水段扩散距离为 $23 \mathrm{~cm}$, 实测数据和数值模 拟结果的误差不大.

\section{5 注浆堵水防渗效果回归分析}

\section{1 回归模型建立}

对本次试验的数据进行多元线性回归分析，定量 分析试验参数分别与注浆后的堵水率和固结体 $1 \mathrm{~d}$ 渗 透系数的函数关系. 由试验分析可知, 试验结果与影响 因素之间并非线性关系, 而大致呈幂函数的关系, 设其 基本模型为

$$
Y=A \cdot X_{1}^{B} \cdot X_{2}^{C} \cdot X_{3}^{D} \cdot X_{4}^{E},
$$

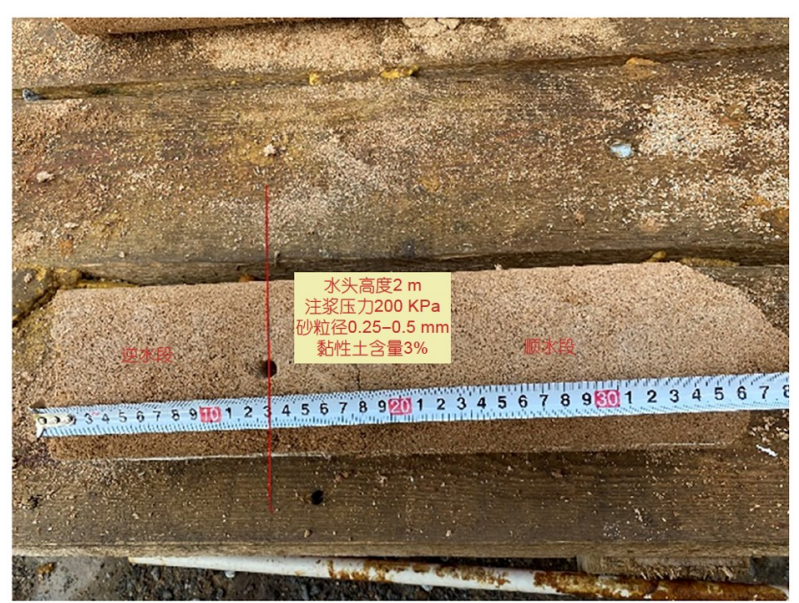

图 19 脱模后固结体

Figure 19 Solidified body after demoulding.

式中, $X_{1}$ 为水头高度, $X_{2}$ 为注浆压力, $X_{3}$ 为砂层粒径, $X_{4}$ 为黏性土含量.

令 $y=\lg Y, a=\lg A, x_{1}=\lg X_{1}, x_{2}=\lg X_{2}, x_{3}=\lg X_{3}, x_{4}=\lg X_{4}$, 可将上述模型化为线性回归模型, 即 $y=a+B x_{1}+C x_{2}+D x_{3}$ $+E x_{4}$.

\section{2 回归方程}

首先对表3中的注浆因素的量值和试验结果值进 行对数转换, 砂层粒径按平均颗粒直径计算, 然后将换 算后的数据在SPSS 26 统计分析软件中进行分析, 求得 的回归系数如表 6 所示.

由表 6 可得到注浆的堵水率 $S E$ 、固结体 $1 \mathrm{~d}$ 的渗透 系数 $K$ 与动水压力 (水头高度) $H$ 、注浆压力 $P$ 、砂层平 均粒径 $D_{\mathrm{P}}$ 、黏性土含量 $M$ 之间的回归方程式为

$$
\begin{aligned}
& S E=75.336 H^{0.04} P^{0.4} D_{\mathrm{P}}^{0.13} M^{0.01}, \\
& K=80.168 H^{-0.04} P^{-0.502} D_{\mathrm{P}}^{0.01} M^{0.16} .
\end{aligned}
$$

堵水率与固结体渗透系数的 $R^{2}$ 均大于 0.9 , 说明回 归拟合效果良好. 从公式中的自变量对因变量的相关

表 6 多元线性回归系数

Table 6 Multiple linear regression coefficients

\begin{tabular}{cccccccc}
\hline 回归系数 & $a_{1}$ & $A_{1}$ & $B_{1}$ & $C_{1}$ & $D_{1}$ & $E_{1}$ & $R_{1}{ }^{2}$ \\
\hline 数值 & 1.877 & 75.336 & 0.04 & 0.40 & 0.13 & 0.01 & 0.939 \\
\hline \hline 回归系数 & $a_{2}$ & $A_{2}$ & $B_{2}$ & $C_{2}$ & $D_{2}$ & $E_{2}$ & $R_{2}{ }^{2}$ \\
\hline 数值 & 1.904 & 80.168 & -0.04 & -0.502 & 0.01 & 0.16 & 0.954 \\
\hline
\end{tabular}


系数可知, 注浆堵水率与注浆压力、砂层粒径有较强 的相关性, 水头高度和黏性土含量的相关系数小, 说 明相关性较小; 固结体 $1 \mathrm{~d}$ 的渗透系数与注浆压力、黏 性土含量有较强的相关性, 水头高度和砂层粒径的相 关系数小, 说明相关性较小.

\section{6 结论}

(1) 渗透型高聚物具有较好的堵水效果, 黏度较 小, 双组分接触后迅速反应生成不透水的固结体, 可 较好地充填与砂层颗粒间的孔隙, 富水砂层经渗透型 高聚物注浆后，10 min内堵水率可达到 $90 \%$ 以上， $1 \mathrm{~d}$ 渗透系数可达到 $10^{-7} \mathrm{~cm} / \mathrm{s}$ 数量级, 达到了理想的堵水 防渗效果.

（2）注浆压力是控制富水砂层注浆堵水防渗效果 的主控因素, 随着注浆压力的增大, $10 \mathrm{~min}$ 内堵水率逐
渐增大、砂层 $1 \mathrm{~d}$ 渗透系数显著降低.

(3) 孔隙水压力计可以实时监控注浆前后模拟砂 层各点处的孔隙水压力变化, 根据各点处孔隙水压力 变化可预知浆液反应持续进行的时间和反应结束后各 点处的孔隙水压力值.

（4）通过数值模拟分析，发现注浆后顺水段浆-水 两相渗流压力场整体表现为从注浆孔向出水边界递 减, 逆水段变化幅度没有顺水段大; 渗流速度场顺水段 渗流的速度在注浆压力和水流冲刷助力作用下要明显 比逆水段的速度大; 存在逆水扩散的现象, 逆水段浆液 扩散距离在水冲刷作用下要明显小于顺水段, 模拟的 结果与注浆后固结体扩散距离实测结果误差不大.

(5) 通过多元线性回归分析, 得到注浆堵水率、固 结体 $1 \mathrm{~d}$ 的渗透系数与动水压力(水头高度)、注浆压力、 砂层粒径、黏性土含量的回归方程，对研究渗透型高聚 物在富水砂层中堵水的规律变化有一定的参考价值.

\section{参考文献}

1 Shao K. Research on measures to deal with water gushing and sand gushing in shield tunnel doors of water-rich sand layer (in Chinese). Shanxi Architect, 2012, 38: 88-189 [郡可. 富水砂层盾构隧道洞门涌水涌砂处理措施研究. 山西建筑, 2012, 38: 188-189]

2 Sun L Y, Huang Y L, Yin C F, et al. Study on the disturbance mechanism of deep hole slurry injection in shallow buried subway tunnel with water-rich sand layer (in Chinese). Modern Tunnel Tech, 2018, 55: 184-193 [孙连勇, 黄永亮, 尹长凤, 等. 富水砂层浅埋地铁隧道深孔注浆扰 动机理研究. 现代隧道技术, 2018, 55: 184-193]

3 Li S, Liu R, Zhang Q, et al. Protection against water or mud inrush in tunnels by grouting: A review. J Rock Mech Geotech Eng, 2016, 8: 753766

4 Zheng Q. Study on the effect of superfine cement-water glass slurry in water-rich silt stratum concealed excavation tunnel for grouting and water plugging (in Chinese). Railway Construct, 2021, 61: 51-54 [郑青. 超细水泥-水玻璃浆液在富水粉细砂地层暗挖隧道中的注浆堵水效果研究. 铁道建筑, 2021, 61: 51-54]

5 Ruan W J. Study on some basic properties of grout diffusion and slurry (in Chinese). J Geotech Eng, 2005, 27: 69-73 [阮文军. 注浆扩散与浆液 若干基本性能研究. 岩土工程学报, 2005, 27: 69-73]

6 Wang F M, Li J, Shi M S, et al. Research and application of new technology for embankment dam impermeability reinforcement (in Chinese). J Hydropower, 2016, 35: 1-11 [王复明, 李嘉, 石明生, 等. 堤坝防渗加固新技术研究与应用. 水力发电学报, 2016, 35: 1-11]

7 Du X, Fang H, Wang S, et al. Experimental and practical investigation of the sealing efficiency of cement grouting in tortuous fractures with flowing water. Tunnell Undergr Space Tech, 2021, 108: 103693

8 Liu J, Liu R T, Zhang X, et al. Model test and numerical simulation of cement slurry fracture grouting diffusion law (in Chinese). J Rock Mech Eng, 2012, 31: 2445-2452 [刘健, 刘人太, 张霄, 等. 水泥浆液裂隙注浆扩散规律模型试验与数值模拟. 岩石力学与工程学报, 2012, 31: 2445-2452]

9 Liu Q, Tan Z S, Wang X Y. Model experimental study on the distribution law of seepage field in underwater tunnels (in Chinese). J Civil Eng, 2015, 48: 388-392 [刘强, 谭忠盛, 王秀英. 水下隧道渗流场分布规律的模型试验研究. 土木工程学报, 2015, 48: 388-392]

10 Zhang L Z, Zhang Q S, Zhang X, et al. Study on the diffusion mechanism of infiltration grouting under dynamic water conditions (in Chinese). Modern Tunneling Tech, 2017, 54: 74-82 [张连震, 张庆松, 张霄, 等. 动水条件下渗透注浆扩散机理研究. 现代隧道技术, 2017, 54: 74-82]

11 Hui B, Zhang Q S, Zhang L, et al. Characteristics of the seepage field of high porosity sandstone gushing water and grouting management (in 
Chinese). J Coal, 2014, 39: 184-190 [惠冰, 张庆松, 张立, 等. 高孔隙率砂岩涌水渗流场特征与注浆治理. 煤炭学报, 2014, 39: 184-190]

12 Ning B, Wu S Q, Xie X H, et al. Experimental study on the effect of seepage action on the grouting of sand and gravel layer (in Chinese). Geotech Mech, 2011, 32: 285-289 [宁博, 吴时强, 谢兴华, 等. 渗流作用对砂砾石层灌浆的影响试验研究. 岩土力学, 2011, 32: 285-289]

13 Krizek R J, Perez T. Chemical grouting in soils permeated by water. J Geotech Eng, 1985, 111: 898-915

14 Zhou Z, Du X, Wang S, et al. Micromechanism of the diffusion of cement-based grouts in porous media under two hydraulic operating conditions: Constant flow rate and constant pressure. Acta Geotech, 2019, 14: 825-841

15 Wang Q, Wang S, Sloan S W, et al. Experimental investigation of pressure grouting in sand. Soils Found, 2016, 56: 161-173

16 Bouchelaghem F, Vulliet L, Leroy D, et al. Real-scale miscible grout injection experiment and performance of advection-dispersion-filtration model. Int J Numer Anal Meth Geomech, 2001, 25: 1149-1173

17 Zhou Z, Cai X, Du X, et al. Strength and filtration stability of cement grouts in porous media. Tunnell Undergr Space Tech, 2019, 89: 1-9

18 Axelsson M, Gustafson G, Fransson Å. Stop mechanism for cementitious grouts at different water-to-cement ratios. Tunnell Undergr Space Tech, 2009, 24: 390-397

19 Yu X Y, Zhang N, Zhang H W, et al. Research on polymer chemical grouting material for treating large scale roofing in roadway (in Chinese). Coal Mine Safety, 2010, 41: 10-13 [于宪阳, 张农, 张海韦, 等. 高分子化学注浆材料处理巷道大范围冒顶研究. 煤矿安全, 2010, 41: 10-13]

20 Dai G Z. New Technology of Geotechnical Slurry Materials and Hole Protection Mud (in Chinese). Chongqing: Chongqing University Press, 2015. 193 [代国忠. 岩土工程浆材与护孔泥浆新技术. 重庆: 重庆大学出版社, 2015. 193]

21 Wang Z Q. Research and application of seepage prevention and reinforcement technology for undesirable geological bodies under high head (in Chinese). J Changjiang Acad Sci, 2018, 35: 1-6 [汪在芹. 高水头下不良地质体防渗补强技术研究与应用. 长江科学院院报, 2018, 35: 1-6]

22 Van P L A, Whiffin V S, Harkes M P. Immobilisation of bacteria to a geological material. US Patent. US20060097135, 2009-08-27

23 Dejong J T, Soga K, Kavazanjian E, et al. Biogeochemical processes and geotechnical applications: Progress, opportunities and challenges. Géotechnique, 2013, 63: 287-301

24 Xie D Y, Chen C L, Hu Z Q. Experimental Geotechnical Engineering (in Chinese). Beijing: Higher Education Press, 2011 [谢定义, 陈存礼, 胡再 强. 试验土工学. 北京: 高等教育出版社, 2011]

25 Song X J, Lu T H, Li B W. Discussion on the calculation method of average particle size of sand samples (in Chinese). Riverhead Univ (Nat Sci Ed), 2008, 36: 121-124 [宋新江, 卢廷浩, 李炳蔚. 砂样平均粒径计算方法探讨. 河海大学学报(自然科学版), 2008, 36: 121-124]

26 Qian Z W, Jiang Z Q, Cao L W, et al. Experimental study on infiltration grouting model for weakly cemented pore media (in Chinese). Geotechnics, 2013, 34: 139-143 [钱自卫, 姜振泉, 曹丽文, 等. 弱胶结孔隙介质渗透注浆模型试验研究. 岩土力学, 2013, 34: 139-143] 


\title{
Experiment and numerical simulation of water plugging law of permeable polymer in the water-rich sand layer
}

\author{
FANG HongYuan ${ }^{1,2,3}$, LIU Kang ${ }^{1}$, DU XueMing ${ }^{1,2,3}$ \& WANG FuMing ${ }^{2,3}$ \\ ${ }^{1}$ School of Water Resources Science and Engineering, Zhengzhou University, Zhengzhou 450001, China; \\ ${ }^{2}$ National Local Joint Engineering Laboratory of Major Infrastructure Inspection and Repair Technology, Zhengzhou 450001, China; \\ ${ }^{3}$ Collaborative Innovation Center of Water Resources and Transportation Infrastructure Safety Protection in Henan Province, Zhengzhou 450001, \\ China
}

When constructing a tunnel through a water-rich sand layer, permeation grouting is one of the important methods for preventing seepage and plugging water. Unlike typical chemical slurries, permeable polymers have the characteristics of two-component reaction and rapid consolidation throughout the slurry diffusion process, making them suited for grouting and plugging water in water-rich sand layers. However, due to the characteristics of rapid consolidation in the diffusion process, the diffusion law in a water-rich sand layer is more complex. To study the water plugging effect of this permeable polymer in the water-rich sand layer, a set of visualized permeable grouting model test devices for the water-rich sand layer was created, which has the functions of observing the slurry diffusion distance, monitoring the pore water pressure change before and after grouting, and measuring the water plugging rate. Using the water plugging rate and permeability coefficient as performance indexes to evaluate the water plugging and anti-seepage effects of seepage grouting in a water-rich sand layer and four factors of sand layer particle size, hydrodynamic pressure, grouting pressure, and cohesive soil content as influencing factors of seepage grouting, the orthogonal test of water plugging and anti-seepage effects of seepage grouting in a sand layer is carried out. The main controlling factors affecting the effect of sand layer infiltration grouting are obtained. Simultaneously, the numerical simulation of the model test was carried out, focusing on the change process of pressure, flow rate and slurry diffusion in the slurry-water two-phase seepage field during the grouting process, and the comparison with the experimentally measured slurry diffusion distance was verified. The results show that: (1) after the water-rich sand layer is grouted with permeable polymer, the water plugging rate can reach more than $90 \%$ within $10 \mathrm{~min}$, and the permeability coefficient within a day can reach the order of $10^{-7} \mathrm{~cm} / \mathrm{s}$, achieving the ideal water plugging and seepage control effect. (2) Within a certain range of grouting pressure, the grouting pressure is the primary control factor for controlling the water plugging and anti-seepage effects of grouting in water-rich sand layers. The water plugging rate increases within $10 \mathrm{~min}$ as the grouting pressure increases, and the permeability coefficient of the sand layer within a day decreases significantly. (3) When the slurry reaction is complete, the pore water pressure in the part of the sand layer where the slurry can diffuse is close to zero. (4) The numerical simulation results show that the pressure field of slurry-water two-phase seepage decreases from the grouting hole to the water discharge boundary, the seepage velocity of the downstream section is significantly larger than that of the upstream section, the slurry has the phenomenon of backwater diffusion, and the diffusion distance simulation results have little error with the measured results of the consolidation body. (5) The regression equations of grout plugging rate, $1 \mathrm{~d}$ permeability coefficient of consolidation body with dynamic water pressure, grouting pressure, sand layer grain size, and clay content were obtained by multiple linear regression analysis.

water-rich sand layer, permeation grouting, permeable polymer, model test, numerical simulation, water plugging doi: 10.1360/SST-2021-0461 